\title{
EVALUACIÓN DE LAS COMPETENCIAS EN LOS ESTUDIANTES, URACCAN-NUEVA GUINEA
}

Eugenio López Mairena ${ }^{[1]}$ José Felipe Trillo Alonso[ ${ }^{[2]}$

\section{Resumen}

Esta investigación abordó la evaluación de las competencias en la educación universitaria desde el enfoque teórico como actuaciones integrales para identificar, interpretar, argumentar y resolver problemas del contexto con idoneidad y ética, integrando el saber ser, el saber conocer y el saber hacer, acordes con el acontecer de su entorno sociocultural. La investigación fue documental y en un nivel descriptivo.

Los resultados orientaron realizar transformación curricular desde la perspectiva del enfoque educativo de sistemas complejos interdisciplinares y mutidisciplinares para transversalizar como eje la evaluación de las competencias en los estudiantes en todas las áreas de las ciencias educativas, investigativas, de extensión y gestión institucional.

Palabras clave: Evaluación de las competencias, Currículum y Modelo pedagógico de la URACCAN.

\section{Summary}

This research analyzed the skills evaluation in higher education from the theoretical approach in order to identify, interpret, argue and resolve problems from a suitable and ethical context, integrating the different levels of learning, such as: the know-to be, the know-to know, and the know-to make, that should be consistent with the events of their socio-cultural environment. The research was documentary with a descriptive level.

The results suggested to make curricular changes from the perspective of the educational approach based on the complexity of interdisciplinary and multidisciplinary systems to use the student's skills evaluation as a transversalize axis in all the areas of science: education, investigation, extension and institutional management.

Keywords: Skills evaluation, curriculum and pedagogical model of URACCAN.

[1] Master en Matemáticas. Vicerrector de la URACCAN, Nueva Guinea. Correo electrónico: elmsmp2oo2@yahoo.es

[2] Dr. Profesor de la Universidad de Santiago de Compostela, España. Correo electrónico: felipe.trillo@usc.es 


\section{Introducción}

Actualmente, en el conclave de la Declaración Mundial sobre Educación Superior en el siglo xxi: Visión y acción (Unesco, 1998), se plantea: "La Educación Superior comprende todo tipo de estudios de formación educativa hacia la excelencia para la investigación en el nivel post secundario...". En consecuencia, la Educación Superior debe fundamentarse en el conocimiento y en la investigación, en la mejora de su calidad, en su pertinencia y relevancia.

En el entorno inmediato, la URACCAN es protagonista en la promoción de la Interculturalidad en la Educación Superior en la Costa Caribe, realza esta temática en América Latina y forma parte de la Declaración de la Conferencia Regional de Educación Superior en América Latina. La Universidad se ha constituido en el instrumento estratégico para consolidar el proceso de Autonomía de la Costa Caribe que garantiza la igualdad en la diversidad. En tal virtud converge la necesidad de transversalizar la evaluación de las competencias en los estudiantes desde un lanzamiento hacia la excelencia académica.

Aprender a conocer, aprender a hacer, aprender a convivir se convierten en tres pilares de la educación para hacer frente a los retos del siglo xxI y facilitar desde el personal docente, el escenario y los medios para que cada estudiante se oriente a descubrir, despertar e incrementar su creatividad, permitiéndole transformar los diversos contextos socioculturales en su expresión constructivista, problematizadores, pertinentes, plurisignificativos, técnicos, científicos y humanistas.

Ante el paradigma de complejidad desde el saber de interculturalidad, multiculturalidad y transculturalidad para abordar la realidad desde su pertinencia concreta hasta las situaciones más globalizantes, se hace necesario sérselas en el marco de los Derechos Humanos y facilitar diálogos con pertinencia en dirección estratégica hacia las transformaciones curriculares en lineamientos sistémicos desde la Educación Primaria hasta la Educación Superior en lo que concierne a la evaluación de las competencias en los estudiantes y la necesidad de cumplir con los estándares internacionales de calidad.

\section{Propósito}

Promover estrategias educativas de transformación en el currículum de la URACCANNueva Guinea, generando la oportunidad al diálogo de una comunidad educativa crítica que garantice el desarrollo endógeno y autosostenible desde la perspectiva de la investigación científica, social y tecnológica para constituirse en agente efectivo del cambio y desarrollo social. En la comprensión de que en Nicaragua la evaluación de las competencias en los estudiantes vendrá a responder a la calidad exigida por el Consejo Nacional de Educación Superior (CNU), organismo planificador, regulador y coordinador del Sistema Nacional de Educación Superior de Nicaragua. 
La evaluación de las competencias en los estudiantes desde la perspectiva de la Autoevaluación Institucional con Fines de Mejora tiene su fundamento en las disposiciones de la legislación nicaragüense que regulan la educación del país, y que están contenidas en la Constitución Política de la República, en la Ley General de Educación (Ley 582), en la Ley de Autonomía de las Instituciones de Educación Superior (Ley 89) y en la Ley Creadora del Sistema de Evaluación y Acreditación para el Aseguramiento de la Calidad de la Educación y Reguladora del CNEA (Ley 704).

En esta Ley 704 se establece que cada una de las instituciones de Educación Superior de Nicaragua, realizarán procesos de autoevaluación institucional, que les permita identificar sus fortalezas, debilidades y formular planes de mejora que garanticen la calidad de la educación. Esta investigación documental tiene importancia en los procesos de autoevaluación universitaria y comprenderá todos aquellos factores que la URACCAN establece en su visión y misión. En tal razón la evaluación de las competencias en los estudiantes deviene en paradigma que responde a los desafíos del S. XXI en referencia a la excelencia en la Educación Superior.

\section{Revisión de literatura}

En el ámbito educativo existe una amplia literatura sobre la evaluación, esta se enfoca en la evaluación de las competencias en los estudiantes a partir de la complejidad y el currículum divergente en tanto permite la valoración entre teoría y práctica educativa. Esta dimensión del currículum es la que se vincula con dos temas de vigencia en la actualidad: La visión del profesor como un profesional reflexivo y la investigación como proceso para su formación y desarrollo profesional.

\section{Concepto}

Competencia, según la Real Academia Español (RAE) proviene del lat. competentǐa; cf. Competir. Aunque el término es polivalente en la significación en cuanto hace alusión en primer lugar a: Disputa o contienda entre dos o más personas rivales sobre algo. También comprende desde la palabra incumbencia hasta la significación de pericia, aptitud, idoneidad para hacer algo o intervenir en un asunto determinado.

El concepto de competencia del que partimos es bien sencillo. Lo adoptamos del denominado proyecto Tuning, según el cual las competencias son: "Conjunto de conocimientos, de habilidades y actitudes que se aplican en el desempeño de una profesión”.

\section{El concepto de competencias orientado a la formación integral:}

La propuesta innovadora más relevante de todas las surgidas al albur del EEES, que es la que tiene que ver con la promoción del aprendizaje autónomo mediante la adopción de un modelo de enseñanza centrado en el estudiante. "la capacidad para 
tomar decisiones argumentadas y ensayar alternativas para resolver problemas complejos en contextos de incertidumbre" (Trillo, 2010d).

\section{Materiales y métodos}

La investigación documental, variante de la investigación científica, cuyo propósito es el análisis de diferentes fenómenos, utiliza técnicas precisas para alcanzar los resultados de valoración, así los aportes teóricos que reúnan las cualidades de integridad e importancia, delimitadas a las formas de orden metodológico, a fin de dar respuestas a las interrogantes de la investigación. La originalidad del estudio se refleja en el enfoque, criterios, conceptualizaciones, conclusiones, recomendaciones y en general el pensamiento del autor.

\section{Nivel de la investigación}

El nivel de la investigación representa la obtención de la información acerca de un fenómeno o proceso, para describir sus implicaciones, intereses y profundidad con que se emprende un objetivo, lo cual aquí se señalara si se refiere a una investigación exploratoria, descriptiva o explicativa.

Esta investigación es descriptiva. Su meta no se limita a la recolección de datos, sino a la predicción e identificación de las relaciones que existen entre dos o más variables y establecer comparaciones con otras fuentes para precisar proyecciones.

\section{Paradigma de la investigación}

Se enfoca desde la fenomenología simbólica y filosofía científica por cuanto son ciencias complementarias desde una teoría interpretativa, dinámica, múltiple, holística, construida y divergente. Pretende comprender e interpretar la realidad, los significados del individuo, las percepciones, intenciones y acciones en contextos socioculturales problematizadores.

\section{Diseño de la investigación}

La investigación tiene un diseño bibliográfico, el cual está basado en la revisión metódica y rigurosa de material documental relacionado al estudio.

\section{Resultados y conclusiones}

En el sistema de evaluación de las competencias en los estudiantes se ha generado la ruptura histórica y epistemológica entre sistema de evaluación tradicional y el nuevo paradigma basado en la evaluación a partir de la globalización y problemáticas complejas. 
No se reproduce lo que otros autores han aportado en la observación a las malas prácticas en la evaluación de los estudiantes (Santos Guerra, 1993; Blanco Prieto, 1994; Santos Guerra,1999; Trillo y Porto, 1999; Porto, 2005; Tobón, et al., 2006; Trillo, 2010d), o a la orientación de lo que debería hacerse (Biggs, 2005; De Miguel M. , 2006; Pérez Gómez, 2008; Ángulo, 2008; Trillo, 201ob; 2010d) en función de los objetivos formativos y las peculiaridades de los contenidos de cada tipo de estudios (nivel, campo de conocimiento, la titulación, etc.).

\section{Evaluación de las competencias en los estudiantes}

En el siguiente cuadro se sintetiza lo que diferentes autores consideran como características de la evaluación de las competencias.

Cuadro No. 1. Características de la evaluación por competencias educativas

\begin{tabular}{|c|l|}
\hline & -Democrática: La verdad y la valoración es asunto de construcción democrática de todos, por lo que en su búsqueda no puede \\
& haber más que participantes. \\
& -No es el evaluador el que juzga, sino que facilita que sean los implicados los que lo hagan. \\
& -No pretende convertir las complejidades naturales en simplificaciones que falsifiquen la realidad, con la justificación de que \\
& simplificando es más fácil establecer conclusiones o emitir juicios (lo que no es del todo cierto). \\
Angulo & -Aunque puede utilizar mediciones y procedimientos estadísticos de análisis de datos, no pretende caer en la mística de los \\
Rasco, & números o de las estadísticas. \\
Contreras & -Se siente comprometido con la utilización de procedimientos metodológicos sensibles a la riqueza, con procedimientos sufi- \\
Domingo & cientemente sensibles como para captar las valoraciones, aspiraciones, interpretaciones e intereses de las personas tal como son \\
$\mathbf{\&}$ & expresadas por ellas mismas, sin presuponerlos de antemano. \\
Santos & -Parte de la clara explicitación de los criterios éticos que conformarían los límites de su desarrollo. \\
Guerra & -Tiene como objetivo material la publicidad de sus informes, y que rechaza cualquier imposición para mantenerlos en secreto o \\
$\mathbf{( 1 9 9 1 )}$ & el ocultamiento de información valiosa. \\
& -Cuida que el lenguaje de sus informes sea accesible no sólo a los directamente implicados en la realidad evaluada, sino a \\
& cualquier otro ciudadano interesado en el conocimiento de dicha realidad. \\
& -Se pretende educativa: Porque se pregunta por el valor educativo del programa y porque busca favorecer la reflexión y el debate \\
de los implicados y su aprendizaje a partir de su propia experiencia. \\
\\
-Reconoce que un informe de evaluación puede crear una estructura social de relación y participación. \\
-Se siente comprometida con la sociedad en general y con el desarrollo y la profundización de la democracia política.
\end{tabular}




\begin{tabular}{|c|c|}
\hline $\begin{array}{l}\text { Blanco } \\
\text { Prieto } \\
\text { (1994) }\end{array}$ & $\begin{array}{l}\text {-Integral: Dirigiéndose a los tres tipos de contenidos curriculares. } \\
\text {-Integrada: Incluyéndose en el proceso general de enseñanza-aprendizaje. } \\
\text {-Integradora: Atendiendo al desarrollo integral del alumno. } \\
\text {-Criterial: Determinando la situación de los alumnos en relación a unos criterios perfectamente definidos y no en relación con el } \\
\text { resto de sus compañeros. } \\
\text {-Educativa: No sólo porque debe mejorar el objeto evaluado, sin también porque debe favorecer el aprendizaje de sus partici- } \\
\text { pantes. } \\
\text {-Total: Dirigiéndose no sólo a aprendizajes de los alumnos, sino también al profesorado, procesos de enseñanza, planificación } \\
\text { curricular, etc. } \\
\text {-Cientíica: Demandando por tanto formación adecuada a quien debe realizar tareas evaluadoras. } \\
\text {-Contextualizada: Atendiendo a todo el contexto en el que está inserto el objeto de evaluación. } \\
\text {-Negociada: En la medida en que un sujeto evaluado participe de la evaluación se sienta coautor del modelo y comparta los } \\
\text { informes, mayores son las garantías de que la evaluación discurra por cauces adecuados y no se desborde anegando todas las } \\
\text { expectativas. } \\
\text {-Ética: Sinceridad, colaboración, respeto, diálogo, intereses "nobles", intencionalidad formativa, tratamiento objetivo, profe- } \\
\text { sionalidad, confidencialidad, rechazo a intereses espurios, cumplimiento de compromisos, etc., deben ser pautas que rijan el } \\
\text { proceso evaluativo. } \\
\text {-Clara: El nivel de precisión terminológica ha de alcanzarse simultáneamente al logro del máximo grado de comprensión, } \\
\text { utilizando un lenguaje claro y directo que sea entendido por todos. } \\
\text {-Participativa: La evaluación debe ser un trabajo cooperativo, colegiado y colaborativo, favoreciendo la implicación de todos en } \\
\text { la elaboración del diseño, durante el proceso y en la fase final de redacción y difusión del informe de evaluación. }\end{array}$ \\
\hline $\begin{array}{c}\text { Blanco } \\
\text { Felip } \\
\text { (1996) }\end{array}$ & $\begin{array}{l}\text {-Procesual: Forma parte intrínseca del mismo aprendizaje. } \\
\text {-Integral: Abarca todos los elementos de intervención. } \\
\text {-Sistemática: Se produce en la misma actuación docente. } \\
\text {-Continua. El proceso no puede evaluarse en momentos aislados, sino en su propia evolución. } \\
\text {-Estructurante: Permite ajustar las actuaciones del proceso de enseñanza-aprendizaje al desarrollo personal y social del alumno. } \\
\text {-Progresiva: Tiene siempre en cuenta de manera intrínseca, el crecimiento, los logros, la madurez y el desarrollo alcanzado por } \\
\text { el alumno. } \\
\text {-Innovadora: Constata siempre los factores que inciden en el proceso de enseñanza-aprendizaje y busca constantemente la } \\
\text { toma de decisiones nuevas. } \\
\text {-Científica: Analiza todos los elementos del proceso como partes de un sistema, con el fin de determinar cuál es el papel de } \\
\text { cada uno de ellos dentro de aquél. }\end{array}$ \\
\hline $\begin{array}{c}\text { Santos } \\
\text { Guerra } \\
\text { (1998) }\end{array}$ & $\begin{array}{l}\text {-Está atenta a los procesos y no sólo a los resultados. } \\
\text {-Da voz a los participantes. } \\
\text {-Utiliza métodos diversos y sensibles para explorar la realidad. } \\
\text {-Está encaminada a la mejora de la institución universitaria y, a través de ella, de la sociedad. } \\
\text {-Es educativa. } \\
\text {-Tiene en cuenta los valores. } \\
\text {-Es holística. } \\
\text {-Nadie tiene el privilegio de la verdad. } \\
\text {-Es democrática. } \\
\text {-Constituye una ayuda y no una amenaza. } \\
\text {-Está contextualizada. } \\
\text {-Utiliza el lenguaje natural de los protagonistas para expresarse. } \\
\text {-Es emergente. }\end{array}$ \\
\hline
\end{tabular}




\begin{tabular}{|c|c|}
\hline $\begin{array}{c}\text { Barberá } \\
\text { (1999) }\end{array}$ & $\begin{array}{l}\text {-Coherente: Ha de diseñarse en estrecha relación y de manera paralela al contenido y a } \\
\text { las actividades de aprendizaje. } \\
\text {-Compartida: Ha de incorporar progresivamente la actuación conjunta de profesores y } \\
\text { alumnos. } \\
\text {-Contextualizada: Las actividades de evaluación han de ser significativas para los alumnos } \\
\text { y lo más reales posibles. } \\
\text {-Continua: Requiere disponer de manera permanente de información sobre el proceso de } \\
\text { aprendizaje del alumno. } \\
\text { - Criterial: Debe tener unos criterios de referencia para determinar el nivel de desarrollo de } \\
\text { las capacidades propuestas. } \\
\text {-Dialogada: Supone establecer un diálogo continuado entre evaluadores y evaluados. } \\
\text {-Diversificada: Se llevará a cabo utilizando instrumentos diferentes, porque también son } \\
\text { diferentes las capacidades y los tipos de contenidos que se deben evaluar. } \\
\text {-Formativa: Ha de dotarse de mecanismos que adecuen progresivamente la práctica do- } \\
\text { cente y los resultados parciales de los alumnos, con el fin de ajustar el proceso didáctico a } \\
\text { las necesidades que se manifiesten. } \\
\text {-Integral: Ha de contemplar los tres tipos de contenidos. } \\
\text {-Integrada: Ha de estar insertada en el proceso de enseñanza y aprendizaje desde su ini- } \\
\text { cio hasta el final. } \\
\text {-Integradora (global). } \\
\text {-Transparente: Ha de ser clara, sobre todo para los alumnos, que son los evaluados. }\end{array}$ \\
\hline $\begin{array}{c}\text { Álvarez } \\
\text { Méndez } \\
\text { (2001) }\end{array}$ & $\begin{array}{l}\text {-Democrática: De forma que en ella participen todos los sujetos que se ven afectados por } \\
\text { la evaluación. } \\
\text {-Está al servicio de quienes son los protagonistas en el proceso: Constituyéndose en re- } \\
\text { curso de formación y oportunidad de aprendizaje. } \\
\text {-Negociada: "se negocia todo, desde la justificación de la propia evaluación hasta las } \\
\text { formas en las que se va a llevar a cabo y el papel que cada uno tiene que asumir respon- } \\
\text { sablemente". } \\
\text {-Transparente: garantizando la publicidad y conocimiento de los criterios que se han de } \\
\text { aplicar. } \\
\text {-Procesual: continua, integrada en el currículum y, con él, en el aprendizaje. } \\
\text {-Formativa: motivadora, orientadora. } \\
\text {-Aplica técnicas de triangulación: Hay que considerar su valor como recurso y como ga- } \\
\text { rantía de la coherencia con el planteamiento de partida, la participación del profesor, la } \\
\text { del alumno que se (auto) evalúa y la de los compañeros con los que éste ha trabajado y } \\
\text { que (co)evalúan. Ellos tres constituyen los vértices del triángulo. } \\
\text {-Asume y exige la responsabilidad de todos los implicados: La responsabilidad del profe- } \\
\text { sor está en garantizar que aquello que los alumnos estudian, leen y aprenden merece la } \\
\text { pena que sea objeto de aprendizaje. La de los alumnos, consiste en tomar consciencia de } \\
\text { que ellos son los responsables máximos de su propio aprendizaje. } \\
\text {-Orientada a la comprensión y al aprendizaje, no al examen. } \\
\text {-Centrada más en la forma en que el alumno aprende, sin descuidar la calidad de lo que } \\
\text { aprende. }\end{array}$ \\
\hline
\end{tabular}

Fuente: (Porto 2005, p. 123). 
En ningún momento se pretende incurrir en el error de descalificar de manera general y gratuita. La descripción hecha por el profesor Felipe Trillo (2010d), se refiere a un panorama de la evaluación que se ha caracterizado por:

La desconsideración del alumno, a quien se ve fundamentalmente como un estorbo, una carga que resulta obligatoria atender, eso sí, en el menor tiempo posible, pues distrae de menesteres más interesantes (y rentables) como son la investigación y las publicaciones, la extensión y proyección pública en congresos y similares (consultorías), el gobierno y la gestión académica, etc.

[...] lo que advertimos es que no se pide planificación, ni análisis, ni conjeturas, ni tampoco toma de decisiones. Los alumnos no tienen que explicar lo que hacen ni revisarlo. No se aprecia comprensión. Sólo necesitan recordar conceptos, reproducir ejercicios, organizar la información y representarla (Trillo, 2010d, p. 12).

\section{Evaluación de las competencias en la educación universitaria}

\section{Problemática}

En el contexto actual del nuevo paradigma de la enseñanza basada en competencias, en ese marco, la adaptación a las nuevas metodologías de enseñanza y la inclusión de nuevos enfoques evaluativos centrados en el estudiante son los pilares del proceso de convergencia europea en torno a la Educación Superior en cuanto a la docencia (Gallardo, 2011).

El Espacio Europeo de Educación Superior ha abierto nuevos causes para definir la orientación de los estudios de forma que estos se adecuen a las condiciones y demandas que plantea la sociedad del conocimiento y su consecuencia inmediata de formación a lo largo de la vida (Zabalza, 2005, p. 253).

Planteada la problemática, compleja, con respecto a la evaluación en la Educación Superior, vamos a tomar como punto de partida algunas consideraciones propuestas por Cano (2008). "La evaluación se halla en la 'encrucijada' didáctica, en el sentido de que es efecto pero a la vez es causa de los aprendizajes" (p. 9). Esta autora siguiendo a Barberá (1999), Allen (2000), McDonald et al. (2000), Dochy et al. (2002), Bain (2006), plantea que estos escritores nos inducen a entender que la evaluación:

no puede limitarse a la calificación (sino que esta es un subconjunto de la evaluación); no puede centrarse en el recuerdo y la repetición de información (sino que se deben evaluar habilidades cognitivas de orden superior) y que no puede limitarse a pruebas de 'lápiz y papel', sino que se requieren instrumentos complejos y variados (Cano, 2008, p. 9). 
Desde los enfoques del currículum por competencia se sigue a Le Boterf (2002), Perreneud (2005), Tardif (2006), "un saber actuar complejo que se apoya en la movilización y la combinación eficaz de una variedad de recursos internos y externos dentro de una familia de situaciones". (Tardif, 2006, p. 22), la evaluación de las competencias deberá realizarse en una situación lo más auténtica posible (Lussier y Allaire, 2004). Entendiendo por situación auténtica, una situación lo más cercana posible al contexto profesional en el que los estudiantes habrán de desenvolverse una vez que hayan finalizado sus estudios.

Sucintamente, podemos decir que la evaluación por competencias tiene las siguientes características: 1) Es un proceso dinámico y multidimensional que realizan los diferentes agentes educativos implicados (docentes, estudiantes, institución y la propia sociedad); 2) Tiene en cuenta tanto el proceso como los resultados del aprendizaje; 3) Ofrece resultados de retroalimentación de manera tanto cuantitativa como cualitativa; 4) Tiene como horizonte servir al proyecto ético de vida (necesidades personales, fines, etc.) de los estudiantes; 5) Reconoce las potencialidades, las inteligencias múltiples y las zonas de desarrollo de cada estudiante y 6) Se basa en criterios objetivos y evidencias consensuadas socialmente, reconociendo además la dimensión subjetiva que siempre hay en todo proceso de evaluación, [...] informa sobre las acciones necesarias para superar las deficiencias en las mismas (García, 2005; Tobón, 2005, Tobón et al., 2006).

Si queremos evaluar competencias hemos de contar, por consiguiente, con el diseño de escenarios de evaluación auténtica, de situaciones reales donde poner en práctica las mismas (Gallardo, 2011).

\section{Criterios generales de actuación}

En el marco del proceso de convergencia europea y el Tuning, en América Latina es necesario un cambio de metodología por parte de los docentes para la formación y la evaluación de competencias: Esta metodología, implica una organización de los contenidos y unos sistemas de evaluación acordes con este nuevo modelo educativo (Gallardo, 2011).

Partimos de lo que nos dice Biggs, si realmente deseamos enseñar a los estudiantes "con el fin de que piensen, decidan y actúen en el mundo real... La tarea de evaluación debe requerir en algún momento una demostración activa de su capacidad de poner en acción el conocimiento" (Biggs, 2005, p. 189).

Tomando como principio la afirmación anterior retomamos las siguientes recomendaciones generales que nos hace el profesor Felipe Trillo (2010d): 
1. Asumir lo esencial es emitir un juicio de valor serio y fundado, reflexivo, intencional y sistemático, que es subjetivo, pero jamás arbitrario, frente a la falsa lógica aritmética o el capricho dogmático.

2. Asumir una responsabilidad educativa y primar la función formativa, frente a la sumativa y social acreditativa. En esa dirección es imprescindible que la evaluación se realice durante el proceso de enseñanza-aprendizaje y que tenga por objetivo ayudar a los profesores y estudiantes a regular, de manera interactiva, su capacidad en ese mismo proceso.

3. Asumir que la tarea es compleja y delicada por lo que exige dedicación (tiempo) y tacto, frente a una perspectiva mecanicista y fríamente burocrática.

4. Asumir que es preciso adoptar estrategias que primen, preferentemente, el conocimiento funcional (puesta en acción) y también el divergente (abierto), frente al academicismo especulativo y falsamente unívoco (Biggs, 2005, p. 190-200).

5. Asumir que es necesario plantear, preferentemente, tareas relevantes y contextualizadas, e incluso a medio plazo, frente al academicismo aburrido, abstracto y falsamente automático.

6. Asumir que es obligado valorar las realizaciones desde una perspectiva holística y ética, frente a la disección analítica que ignora que 'el todo es mucho más que la suma de las partes'.

\section{Recomendaciones específicas para la práctica}

Consideremos los contenidos, y para ello tomamos como idea básica de partida: El currículum disciplinar y la obsesión academicista deben substituirse por un currículum basado en problemas y organizado en proyectos de trabajo, interpretación y experimentación (Pérez Gómez, 2001, p. 226, citado en Trillo 2010d, p. 15).

El profesor Trillo extrae algunas consecuencias importantes para la práctica de la evaluación a partir de la cita anterior, la cual considera de interés epistemológico sobre el conocimiento en los procesos formativos:

1. Tener bien presente que un asunto carente de significado no puede promover un aprendizaje motivado y orientado a la comprensión.

2. Esforzarse por proponer tareas relevantes.

3. Reflexionar el interés que las tareas de evaluación suscitan en los alumnos en orden de mantener su motivación, recordando que una cosa es plantear simplezas que les entretengan y otro el dilema que supongan un desafío personal.

4. Cuidar la forma cómo se presentan las tareas, especialmente respecto a la claridad con que se hace de manera que pueda reconocerse su sentido, evitando 
por tanto la ambigüedad y con ello la confusión o el desconcierto a la que a menudo se arrastra a los alumnos.

5. Es fundamental que el estudiante use el conocimiento y no sólo que sepa cosas. Para ello, hay que plantearle principalmente dilemas y sugerirle vías de solución ante los cuales pueda desarrollar procesos de deliberación más que adoptar caminos o soluciones cerradas.

Considerando los instrumentos, y desde la evidencia evaluativa, se orienta:

1. Ubicar definitivamente la evaluación en el proceso mismo de enseñanzaaprendizaje, evitando que se sitúe al final como un momento distinto. La evaluación debe ser continua.

2. Asegurarse de que la estrategia de evaluación adoptada es coherente con la metodología desarrollada en el aula: En esta dirección, existen alternativas didácticas más acertadas para el desarrollo de las competencias, cuya lógica particular hay que respetar. El aprendizaje deberá basarse en problemas, estudio de casos, simulación de toma de decisiones, proyectos interdisciplinares, etc. Y a otro nivel: Seminarios y talleres, clases prácticas, prácticas externas, tutorías, trabajo en grupo, trabajo autónomo, etc.

3. Adoptar una pluralidad de instrumentos y procedimientos metodológicos congruentes con esa finalidad: ensayos, trabajos proyectos, observación, portafolio, entrevistas, exposiciones orales, cuadernos de campo, seminarios de debate y reflexión, rubricas holísticas, entre otras.

4. La evaluación ha de ocupar un lugar central si queremos que progresivamente cada sujeto vaya asumiendo la responsabilidad de conocer y autorregular sus procesos de aprendizaje y actuación (Pérez Gómez, 2008, p. 94; Trillo, 2010d, p. 15-16).

\section{Considerando las condiciones es necesario:}

1. Reflexionar sobre cómo se maneja el tiempo: si es rígido o flexible; si implica tareas cortas o de medio y largo plazo; si han tenido tiempo suficiente para completarla; y si se prevé o no tras finalizar la tarea, un tiempo para su revisión en profundidad (realizar metaevaluación).

2. Preguntarse acerca de si son suficientes los recursos de los que se dispone (basta pensar en la accesibilidad a los medios, pues si ésta no es buena todo se entorpece y retrasa).

3. Considerar si el clima de aula es el indicado: Si el ambiente es sosegado, sereno, amable, optimizador, o si por el contrario resulta inquisitivo, amenazante, precipitado, elevando los niveles de tensión, de ansiedad y hasta de angustia. 
4. Pararse a pensar en las razones que empleamos para decidir que un trabajo se realice de manera individual, incluso competitiva, o en equipo y de forma colaborativa.

5. Considerar autocríticamente cuáles han sido las pautas de interacción que se mantienen entre profesores y alumnos cuando se trata de su evaluación: Si ha sido cordial y de ayuda, aunque exigente, o si ha resultado ser hostil o recelosa y de oposición o distante (Ibíd. p. 16).

\section{Para la evaluación del aprendizaje autónomo}

La idea básica es el lanzamiento con la tarea de reconstrucción crítica del conocimiento mediante la atribución de sentido del que cabe emerja un pensamiento divergente (lo que no quiere decir acertado).

Asumido eso es necesario saber que tal pretensión exige atender preferentemente las capacidades:

1. De la autorregulación y metacognición en el aprendizaje. Comprende y reflexiona acerca del estado mental de sí mismo y de otro. Es la capacidad de prever el comportamiento propio y ajeno, gracias a la percepción de sensaciones, emociones y creencias.

2. De iniciar procesos conducentes a relacionar la información entrante con la experiencia previa a fin de extraer significados personales.

3. De interpelar el conocimiento y contextualizarlo.

4. De formular hipótesis, hacer inferencias y contrastarlas mediante el análisis de las evidencias.

5. De tomar decisiones argumentadas y ensayar alternativas para resolver problemas en contextos de incertidumbre.

6. De autoevaluar los resultados.

De manera más general, el mismo autor en otro trabajo nos sugiere para reforzar esta perspectiva de aprendizaje autónomo y protagonismo del estudiante al atender el desarrollo de ciertas características:

Lo cual, a su vez, exige de los estudiantes que, frente a la posición de mero cliente o paciente, adopten una actitud de estudiante (Trillo, 1999), caracterizada por:

1. La predisposición para asumir una mayor responsabilidad sobre su vida académica.

2. El compromiso con un proyecto personal de profesión.

3. La crítica del conocimiento establecido. 
4. La interrogación y posicionamiento personal en el proceso de aprendizaje.

5. La cooperación entre iguales.

6. La colaboración y demanda en relación con el profesorado.

Para concluir este apartado de la evaluación de las competencias es importante citar la conclusión del profesor Trillo en su conferencia presentada en el Perú: "un estudiante sólo puede abrigar una sólida esperanza en el adecuado desempeño de su futuro profesional, si ha sido capaz de tomar decisiones argumentadas y ensayar alternativas para resolver problemas complejos en contextos de incertidumbre" (Trillo, 2010c, p. 27).

\section{Construcción de evidencias para evaluar competencias}

Para evaluar competencias es necesario recoger evidencias. El proceso de evaluación por competencias se basa en evidencias, es decir en pruebas que los estudiantes aportan durante su proceso de formación con el fin de demostrar el desarrollo de las competencias y sus correspondientes niveles alcanzados (Tobón et al., 2006). Estas evidencias se resaltan en: evidencias de saber, evidencias de actitud, evidencias del hacer, evidencia de producto.

\section{El proceso general de la evaluación de competencia}

En general, el proceso de evaluación de competencias en Educación Superior se compone de cuatro ejes interrelacionados entre sí: evaluación diagnóstica, evaluación formativa, evaluación de promoción y evaluación de certificación. Cada uno de estos ejes de evaluación tiene su particularidad, pero no tienen sentido en sí mismos por que las competencias requieren de todos.

\section{Estrategias para la evaluación}

Las estrategias de evaluación son procedimientos compuestos por un conjunto de pasos orientados a determinar el grado de desarrollo de una determinada competencia o de una o varias dimensiones de ésta. Las estrategias se llevan a cabo con base a instrumentos, los cuales orientan de forma específica la manera de llevarse a cabo la evaluación y permiten obtener la información necesaria sobre el proceso educativo.

Las técnicas de modelaje o ejercicios en la resolución de problemáticas y adquisición de competencias permiten el lanzamiento de las estrategias de evaluación. Algunas de estas se enumeran a continuación: ejercicios en bandeja, ejercicios en grupos, encontrar hechos, ejercicios de escucha, cuestionarios con preguntas cerradas, cuestionarios con preguntas abiertas, evaluación basada en el desarrollo de la actividades, diario reflexivo, construcción de rúbricas para evaluar las competencias. 


\section{Evaluación de las competencias en los estudiantes de la URACCAN}

En este sentido, los diseños curriculares de la URACCAN, sugieren las estrategias de evaluación que deben contener los distintos programas de asignatura.

La evaluación del aprendizaje es continua y debe acompañar el proceso de enseñanza ya que ambos son inseparables, se enmarca dentro de un modelo hermenéuticodialéctico de la educación y de la investigación que da cuenta de las complejidades de los aprendizajes. La evaluación del aprendizaje debe ser un proceso sistemático y permanente que comprende la búsqueda y obtención de información de diversas fuentes acerca de la calidad del desempeño, avance, rendimiento o logro del estudiante y de la calidad de los procesos empleados por el docente, la determinación de su importancia y pertinencia de conformidad con los objetivos de formación que se espera alcanzar (URACCAN, 2007, p. 35).

El proceso de evaluación de aprendizajes del currículo de las carreras de Ciencias de la Educación en: Biología, Español, Matemática e Inglés con Enfoque Intercultural de Género, estará sustentado bajo los siguientes principios: "Evaluar desde la diversidad con perspectiva intercultural de género; evaluar para comprender, evaluar de forma dinámica, evaluar para aprender mejor (URACCAN, 2007, p. 36).

Se puede definir el currículum en evaluación de competencias en aquella estructura que da coherencia a los procesos de enseñanza. Esta dimensión se vincula con dos temas de vigencia actual: La visión del profesor como un profesional reflexivo y la investigación en el aula como proceso para su formación y desarrollo profesional.

\section{Conclusiones}

Existe hoy clara consciencia de que una de las dimensiones de la crisis de los sistemas educativos tiene que ver con la crisis en los modelos hegemónicos de cómo enseñar y sobre todo qué enseñar. El problema es que ya no alcanza con extender la educación, ni tampoco con mejorarla, ahora hay que repensar el modelo y redefinir los tres pilares del triángulo didáctico: Qué se entiende por sujeto de la enseñanza, qué se entiende por sujeto que enseña y qué se entiende por conocimiento 'válido' a transmitir.

\section{El pensamiento sistémico, base de las competencias}

El pensamiento sistémico implica una visión de la realidad compleja en sus múltiples elementos y con sus diversas interrelaciones. Es simplemente el reconocimiento de la naturaleza sistémica del mundo. Observa sus objetos como fenómenos complejos. 
El estilo de pensar que denominamos "pensamiento sistémico" se distingue de otros modos de pensar por varias características (Senge, 1990). Lo esencial de esta nueva forma de pensar consiste en un cambio de enfoque frente al anterior estilo de pensamiento científico, orientado a la explicación de las causas, y que se caracteriza por:

- Observar los problemas en una visión "holística" (en el polo opuesto al reduccionismo metodológico) que haga justicia a las características del campo estudiado sin reducir inadecuadamente esa complejidad.

- Observar relaciones dinámicas en lugar de buscar cadenas lineales de causasefectos.

- Pasar de ver simples "instantáneas" (visión de lo estático) al seguimiento de procesos dinámicos.

- Reemplazar la consideración unidimensional por planteamiento pluridisciplinares.

- Completar el tratamiento analítico de los aspectos cuantitativos con la consideración en visión sintética de los aspectos cualitativos.

- Sustituir el planteamiento determinista, deductivista y cerrado, por planteamientos abiertos, en recursividad circular y apoyados en la creatividad que posibilita la innovación.

- Abandonar el paradigma de la racionalidad calculatoria, que presupone una ontología de lo estable, bien regulado y dominable, con técnicas adecuadas, para ingresar al paradigma de la incertidumbre y la inseguridad ("sociedad del riesgo"; filosofía de la complejidad).

- Pasar del planteamiento de los modelos de "máquina trivial" a los modelos de "máquina no trivial".

Este es el gran campo de reflexión, construcción teórica y práctica que se presenta hoy como desafío a la educación: La construcción de una pedagogía y una didáctica que den cuenta de estas nuevas perspectivas y que permitan generar nuevas herramientas y que correspondan a la viabilidad del currículum por competencias en los procesos de innovación y transformación curricular exigidos como norma de calidad en los estándares de la Educación Superior.

En síntesis, la evaluación orientada a evaluar las competencias en los estudiantes tiene referencia ante problemas del contexto profesional, social, disciplinar e investigativo, estableciendo evidencias e indicadores, buscando determinar el grado de desarrollo en sus tres dimensiones (efectivo-motivacional, cognoscitiva y actitudinal), para brindar retroalimentación en torno a fortalezas y aspectos a mejora en los procesos de metacognición, procesual y actitudinal a partir de la reflexión problematizadora desde la investigación, acción participativa (IAP). 


\section{Recomendaciones}

Valorar críticamente el conjunto de acciones intercientíficas y multidisciplinares en el aspecto cuantitativo y cualitativo en el cambio desde la evaluación tradicional hacia el cambio innovador de las evaluaciones por competencias educativas.

Diagnosticar el estado del arte en relación a la evaluación tradicional y evaluación por competencias educativas, en el recinto URACCAN-Nueva Guinea.

Identificar en cada área del conocimiento las debilidades en el planteamiento de la visión, misión, metas, objetivos, procedimentales y actitudinales en las cuales se necesiten replantearse la evaluación de las competencias educativas.

Realizar transformación curricular desde la perspectiva del enfoque educativo de sistemas complejos interdisciplinares y mutidisciplinares para transversalizar como eje la evaluación de las competencias en los estudiantes en todas las áreas de las ciencias educativas, investigativas, de extensión y gestión institucional.

\section{Lista de referencias:}

Biggs, J. (2005). Calidad del aprendizaje universitario. Madrid: Narcea.

Cano, M. E. (2008). La evaluación por competencias en la Educación Superior. Profesorado. Revista de curriculum y formación del profesorado, 12 (3), 1-16.

García, J. A. (2005). Análisis sociológico de la institución escolar y del conocimiento educativo. En A. Monclus, \& (Coord.), Las perspectivas de la educación actual (págs. 217-226). Salamanca: Témpora.

Le Boterf, G. (2000). Ingeniería de las competencias. Barcelona: Gestión 2000/EPISE.

Gallardo Vigil, M. A. (2011). Evaluación de competencias en la Educación Superior. Un acercamiento teórico. CONHISREMI, Revista Universitaria de Investigación y Dialogo Académico, 7 (2), 1-12.

García Fraile, J.A. et al. (2009). Estrategias didácticas para formar competencias. Lima: AB Representaciones Generales.

Perreneud (2005), Perry, P. (1991). Quality in Higher Education. En T. Schuller, The Future of Higher (págs. 91-99). Bristol: PA: SRHE and Open University Press. 
Pérez Gómez, Á. I. (2008). ¿Competencias o pensamiento práctico? La construcción de los significados de representación y de acción. En J. Gimeno Sacristan, Educar por competencias, ¿qué hay de nuevo? (págs. 59-103). Madrid: Morata.

Porto Currás, M. (2005). La evaluación de los estudiantes universitario: El caso de la Universidad de Santiago de Compostela. Tesis Doctoral . Santiago de Compostela: Universidad de Santiago de Compostela.

Senge, Peter (2010). Quinta disciplina. Disponible en: http://www.alumnos.inf.utfsm. $\mathrm{cl} / \sim$ vpena/ramos/ili26o/textos/La_Quinta_Disciplina.pdf

Real Academia Española. Disponible en línea en: http://lema.rae.es/drae/srv/ search?key=competencia

Tobón, S. (2004). Formación basada en competencias. Bogotá: ECOE.

Trillo, F. (2010). La evaluación de las competencias en la universidad: ¿Qué va antes?

uraccan (2007). Currículo del Profesorado y Licenciatura en Ciencias de la Educación con mención en Español. Regiones Autónomas: Universidad de la Regiones Autónomas de la Costa Caribe Nicaragüense.

UNESCO. Disponible en línea: http://www.unesco.org/education/educprog/wche/ declaration_spa.htm

Zabalza, M. A. (2004). Las cinco miras de la convergencia europea. Recuperado el 18 de Noviembre de 2011, de http://noticias.universia.es/movilidad-academica/ noticia/2004/10/30/610659/cinco-miuras-convergencia-europea.html 